\title{
Prevalence and extent of chronic periodontitis and its risk factors in a Portuguese subpopulation: a retrospective cross- sectional study and analysis of Clinical Attachment Loss
}

\author{
Vanessa Machado ${ }^{1,2}$, João Botelho ${ }^{\text {Corresp., }}{ }^{1,2}{ }^{2}$, António Amaral ${ }^{2}$, Luís Proença ${ }^{2}$, Ricardo Alves ${ }^{1,2}$, João Rua ${ }^{2}$, \\ Maria Alzira Cavacas $^{3}$, Ana Sintra Delgado ${ }^{2}$, José João Mendes ${ }^{2}$ \\ ${ }^{1}$ Department of Periodontology, Clinical Research Unit, Centro de Investigação Interdisciplinar Egas Moniz (CiiEM), Instituto Universitário Egas Moniz, \\ Almada, Portugal \\ 2 Clinical Research Unit, Centro de Investigação Interdisciplinar Egas Moniz (CiiEM), Instituto Universitário Egas Moniz, Almada, Portugal \\ 3 \\ Environmental Health, Centro de Investigação Interdisciplinar Egas Moniz (CiiEM), Instituto Universitário Egas Moniz, Almada, Portugal \\ Corresponding Author: João Botelho \\ Email address: jbotelho@egasmoniz.edu.pt
}

Objectives:To assess the prevalence and extent of chronic periodontitis, and its risk factors in a Portuguese subpopulation referred to periodontal examination.

Methods:This retrospective cross-sectional study used a subset of data from patients who sought dental treatment in a university dental clinic in the Lisbon metropolitan area. The sample consisted of 405 individuals (225 females/180 males), aged 20-90 years. All patients underwent a full-mouth periodontal examination and chronic periodontitis was defined as Clinical Attachment Loss $(C A L) \geq 3 \mathrm{~mm}$ affecting two or more teeth. Aggressive periodontitis cases were excluded from the analysis.

Results:Prevalence of chronic periodontitis was $83.5 \%(95 \% \mathrm{Cl} 80.4-86.6 \%)$. For these subjects, CAL $\geq 3$ $\mathrm{mm}$ affected $86.0 \%$ (95\% $\mathrm{Cl} 84.7-87.2)$ of sites and $83.7 \%(95 \% \mathrm{Cl} 81.7-85.6)$ of teeth, respectively. Mean CAL ranged from 3.6 to $4.3 \mathrm{~mm}$, according to age. In the multivariate logistic regression model, smoking $(\mathrm{OR}=3.55,95 \% \mathrm{Cl} 1.80-7.02)$ and older age $(\mathrm{OR}=8.70,95 \% \mathrm{Cl} 3.66-20.69$ and $\mathrm{OR}=4.85,95 \% \mathrm{Cl}$ 2.57-9.16), for 65+ and 45-64 years old, respectively, were identified as risk indicators for CAL $\geq 3 \mathrm{~mm}$.

Conclusions: This particular Portuguese adult subpopulation a had high prevalence of chronic periodontitis, with severe and generalized clinical attachment loss, and its presence was significantly associated with age and smoking. This data should serve to prepare future detailed epidemiological studies and appropriate public health programs. 


\section{Prevalence and extent of chronic periodontitis and its risk factors in}

2 a Portuguese subpopulation: a retrospective cross-sectional study

3 and analysis of Clinical Attachment Loss

5 Vanessa Machado ${ }^{1,2}$, João Botelho ${ }^{1,2}$, António Amaral $^{2}$, Luís Proença ${ }^{2}$, Ricardo Alves ${ }^{1,2}$, João

$6 \mathrm{Rua}^{2}$, Maria Alzira Cavacas ${ }^{3}$, Ana Sintra Delgado², José João Mendes ${ }^{2}$

$8{ }^{1}$ Department of Periodontology, Clinical Research Unit, Centro de Investigação Interdisciplinar

9 Egas Moniz (CiiEM), Instituto Universitário Egas Moniz, Almada, Portugal

$10{ }^{2}$ Clinical Research Unit, Centro de Investigação Interdisciplinar Egas Moniz (CiiEM), Instituto 11 Universitário Egas Moniz, Almada, Portugal

12 [3Environmental Health, Centro de Investigação Interdisciplinar Egas Moniz (CiiEM), Instituto

13 Universitário Egas Moniz, Almada, Portugal

15 Corresponding author:

16 João Botelho

17 Clinical Research Unit

18 Centro de Investigação Interdisciplinar Egas Moniz (CiiEM)

19 Egas Moniz Cooperativa de Ensino Superior, C.R.L.

20 Campus Universitário, Monte da Caparica, 2829 - 511 Caparica, Almada, Portugal

21 Phone: +351212946737

22 e-mail: jbotelho@egasmoniz.edu.pt.

\section{Abstract}

Objectives: To assess the prevalence and extent of chronic periodontitis, and its risk factors in a Portuguese subpopulation referred to periodontal examination.

Methods: This retrospective cross-sectional study used a subset of data from patients who sought dental treatment in a university dental clinic in the Lisbon metropolitan area. The sample consisted of 405 individuals (225 females/180 males), aged 20-90 years. All patients underwent 
31 a full-mouth periodontal examination and chronic periodontitis was defined as Clinical 32 Attachment Loss $(\mathrm{CAL}) \geq 3 \mathrm{~mm}$ affecting two or more teeth. Aggressive periodontitis cases 33 were excluded from the analysis.

34 Results: Prevalence of chronic periodontitis was 83.5\% (95\% CI 80.4-86.6\%). For these 35 subjects, CAL $\geq 3 \mathrm{~mm}$ affected $86.0 \%$ (95\% CI 84.7-87.2) of sites and 83.7\% (95\% CI 81.736 85.6) of teeth, respectively. Mean CAL ranged from 3.6 to $4.3 \mathrm{~mm}$, according to age. In the 37 multivariate logistic regression model, smoking $(\mathrm{OR}=3.55,95 \%$ CI 1.80-7.02) and older age $38(\mathrm{OR}=8.70,95 \%$ CI 3.66-20.69 and $\mathrm{OR}=4.85,95 \%$ CI 2.57-9.16), for 65+ and 45-64 years old, 39 respectively, were identified as risk indicators for CAL $\geq 3 \mathrm{~mm}$.

40 Conclusions: This particular Portuguese adult subpopulation a had high prevalence of chronic 41 periodontitis, with severe and generalized clinical attachment loss, and its presence was 42 significantly associated with age and smoking. This data should serve to prepare future detailed epidemiological studies and appropriate public health programs.

\section{INTRODUCTION}

Chronic periodontitis is an inflammatory disease characterized by a polymicrobial breakdown of host homeostasis and a progressive destruction of tooth-supporting structures, ${ }^{1,2}$ and its epidemiology and risk factors have been broadly studied. ${ }^{3-6}$

Periodontal diseases have a significant impact on oral health-related quality of life, especially with the worsening and extension of the disease in which it presents higher destructive consequences. ${ }^{7}$ There are important risk factors/indicators for periodontal disease such as alcohol, ${ }^{8}$ overweight and obesity, ${ }^{9}$ smoking ${ }^{6}$ and diabetes. ${ }^{10}$ Also, periodontitis can be a risk factor for several systemic diseases. ${ }^{10-18}$

Some European epidemiological studies have demonstrated the high prevalence of periodontitis among the populations. ${ }^{19-23}$ However, data on the prevalence and risk factors for periodontal disease in the Portuguese population are still missing. According to the latest Portuguese Oral National Health Survey, the prevalence of periodontitis was $10.8 \%$ in adults and $15.3 \%$ in the elderly. ${ }^{24}$ This nationwide survey used the Community Periodontal Index (CPI), with its 
60 recognized limitations. To the best of our knowledge, there are no epidemiological studies that 61 used full-mouth periodontal examination (FMEP) methodology to estimate the prevalence of 62 periodontitis regarding Portuguese samples.

63 The aim of this study was to assess the prevalence, severity, and extent of chronic periodontitis 64 through the full-mouth examination of CAL, and its association with sociodemographic, 65 behavioral and environmental risk factors, in a Portuguese adult subpopulation, of a suburban 66 area of the Lisbon Region, forwarded to periodontal examination.

67

68

69

87

\section{MATERIAL AND METHODS}

The study was conducted in accordance with the Declaration of Helsinki of 1975, as revised in 2013, and approved by the Ethics Committee of Egas Moniz (Ethical Application Ref: 595). A written informed consent was obtained from all participants during the first appointment. After the examination, the participants were informed of their periodontal status, and those with diagnosed periodontal diseases were advised to follow the proper treatment. This protocol followed the STrengthening the Reporting of OBservational studies in Epidemiology (STROBE) guidelines. ${ }^{25}$

\section{Study subjects}

All participants were patients of Egas Moniz Dental Clinic (Almada, Portugal). This university clinic, located in the municipality of Almada, in Setúbal Peninsula (a NUTS III subregion, part of NUTS II Lisbon Region), provides dental health services to the general public.

At the first appointment, patients were submitted to a dental triage protocol, with the application of a self-reported health questionnaire and oral and dental examinations, to guide their treatment needs. Regarding periodontal triage, patients were assessed using the Periodontal Screening and Recording (PSR) procedure ${ }^{26}$, and, if diagnosed with code 2 or higher, they were forwarded to a periodontology appointment.

\section{Patient selection}


89 This retrospective cross-sectional study analyzed patients who attended the dental clinic between

90 September 2015 and March 2017. From a total of 3648 subjects who sought the first consultation

91 in the university dental clinic during that period, 1501 (41\%) patients were referred to the

92 periodontology department, based on their triage status. From these, 459 attended a periodontal

93 consultation and were considered for this study. Fifty-two participants were excluded due to

94 incomplete questionnaires and periodontal data, and two subjects diagnosed with aggressive 95 periodontitis. Hence, a final sample size of 405 subjects was obtained (11\% of the total, $27 \%$ of 96 the patients forwarded for periodontal treatment).

\section{Health questionnaire}

99 Before clinical examinations, all patients answered a general and oral health questionnaire that 100 included information such as age, gender, educational level, employment status, general medical 101 history and medication, smoking status and oral hygiene habits.

102

103

\section{Clinical data}

\section{Periodontal status}

105 Five well-trained and calibrated periodontists (R.A., J.C., C.I., F.J., L.A.) performed all dental and periodontal examinations. Periodontal examinations were performed using CDC/AAP fullmouth periodontal examination (FMEP) methodology. ${ }^{27}$ We defined chronic periodontitis was defined as CAL $\geq 3 \mathrm{~mm}$ affecting two or more teeth. ${ }^{28}$ All permanent fully erupted teeth were examined, excluding third molars, retained roots, and implants. The evaluated parameters were: missing teeth, presence or absence of supragingival biofilm (SB), probing depth (PD), bleeding

111 on probing (BOP), gingival recession (REC) and clinical attachment loss (CAL). SB and BOP

112 were scored on four surfaces of each tooth (mesial, distal, buccal and lingual). At six sites per 113 tooth (mesiobuccal, mid-buccal, distobuccal, mesiolingual, mid-lingual and distolingual), PD

114 was measured as the distance from the cementoenamel junction (CEJ) to the bottom of the 115 pocket and REC as the distance from the CEJ to the free gingival margin, and this assessment 116 was assigned a negative sign if the gingival margin was located coronally to the CEJ. CAL was 
117 calculated as the algebraic sum of PD and REC. It was used a CP-12 SE (Hu-Friedy, Chicago, 118 IL, USA).

119

\section{Measurement reproducibility}

121 Prior to the initiation of the study, all examiners were submitted to theoretical and practical 122 training in a total of ten volunteer non-study patients suffering from moderate to severe 123 periodontitis. The inter-examiner correlation coefficients, at subject level, ranged from 0.76 to 1240.97 and between 0.91 and 0.99 , for mean PD and mean CAL, respectively.

125

126 Covariates

127 Sociodemographic variables and several periodontal disease risk factors were selected as 128 confounding variables. The selected variables were: age, gender, educational level, employment 129 status, smoking status, Body Mass Index (BMI), time elapsed since the last dental appointment, 130 consultation motive and oral hygiene habits.

131 Educational level was assessed as three categories: elementary (1-4 years), middle (5-12 years) 132 and higher ( $>12$ years) education. Employment status of each participant was classified as: 133 employed, unemployed or retired. Smoking status was defined as non-smoker or smoker. Active 134 smokers were further divided into three categories: light smokers $(<10$ cigarettes per day), 135 medium smokers (10-20), heavy smokers $(>20)$. The height of the participants was measured in 136 centimeters, using a hard ruler installed vertically and secured with a stable base. Weight was 137 assessed in kilograms using mechanical scales. BMI was calculated as the ratio of the 138 individual's' body weight to the square of their height. Four BMI categories were defined using 139 WHO criteria ${ }^{29}$ : underweight (BMI < $18.5 \mathrm{~kg} / \mathrm{m} 2$ ), normal weight (BMI 18.5 - $24.9 \mathrm{~kg} / \mathrm{m} 2$ ), 140 overweight (BMI $25-29.9 \mathrm{~kg} / \mathrm{m} 2)$ and obese $(B M I \geq 30 \mathrm{~kg} / \mathrm{m} 2)$. The time elapsed since last 141 dental consult was classified into five categories (never visited, less than one year, 1-2 years, 3-4 142 years, 5 years or over). Consultation motives were classified as routine, aesthetics, pain,

143 functional or other. Oral hygiene habits were assessed by information on toothbrush frequency 144 (2-3 times / daily, one time daily, 2-6 times/weekly) and dental floss use. 


\section{Data analysis}

147 Data analysis was performed using IBM SPSS Statistics version 24.0 for Windows (Armonk,

148 NY: IBM Corp.). Descriptive and inferential statistics methodologies were applied. In the latter,

149 Mann-Whitney and Kruskal-Wallis tests were used to compare the clinical data as a function of

150 the sociodemographic variables. Further, logistic regression analysis was used to model the

151 relationship between chronic periodontitis and several risk indicators. Preliminary analyses were

152 performed using univariate models. Next, a multivariate model was constructed for the outcome

153 variable $\mathrm{CAL} \geq 3 \mathrm{~mm}$. Only variables showing a significance $\mathrm{p} \leq 0.25$ in the univariate model

154 were included in the multivariate stepwise procedure. Predictor variables considered in this

155 procedure were: age (years), smoking status, education (years), employment status, last dental

156 visit and dental floss use. The contribution of each variable to the model was evaluated by Wald

157 statistics. Interactions were also analyzed for all tested variables. The final reduced model was

158 obtained with the following predictor variable categories: age (45-64 and $\geq 65$ years) and

159 smoking status (smoker). Odds ratio (OR) and 95\% confidence intervals (95\% CI) were

160 calculated for both univariate and multivariate analyses. The level of statistical significance was

161 set at $5 \%$.

162

163

164

165

\section{RESULTS}

166 Table 1 shows the distribution of sociodemographic, behavioral, biometric and oral hygiene data 167 in the studied sample. Ages ranged from 20 to 90 years. The sample had 55.6\% of female 168 patients. It is worth to mention that $65.2 \%$ of subjects did not smoke and active smokers were 169 mainly medium smokers (66\%), followed by light smokers (29\%) and heavy smokers $(5 \%)$. 170 Regarding education and employment status $77.1 \%$ of subjects had an elementary or middle 171 level education, and $51.9 \%$ of the subjects were employed. Approximately 59\% were overweight 172 and obese, and only 40\% had normal values. Interestingly, 53.1\% had a period of over one year 173 without any dental visit and 1.2\% never had a dental appointment, whereas functional complaint 174 was the major consultation motive. 
175 Table 2 shows the periodontal data of this sample according to age, gender, and smoking status.

176 Subjects over 65 years of age had a significantly higher mean number of missing teeth and, in

177 total, this subpopulation presented a mean loss of 8 teeth. Younger individuals $(<45$ years of

178 age) presented a significantly lower mean number of missing teeth, PD, REC, furcation lesions

179 and teeth with mobility compared to older subjects. Male patients presented a significantly

180 higher mean PD, deep periodontal pockets $(\geq 5 \mathrm{~mm})$ and teeth with furcation lesions than

181 female. Compared to smokers, non-smokers had lower mean SB, PD and CAL, and less deep

182 periodontal pockets.

183 Chronic periodontitis was diagnosed in $83.5 \%$ of the patients (Table 3), and subjects with

184 chronic periodontitis had CAL $\geq 3 \mathrm{~mm}, \geq 4 \mathrm{~mm}, \geq 5 \mathrm{~mm}, \geq 6 \mathrm{~mm}$ and $\geq 7 \mathrm{~mm}$ affecting, on 185 average, $83.7 \%, 54.4 \%, 32.1 \%, 17.8 \%$ and $9.2 \%$ of their teeth, respectively (Table 4). Besides, 186 the first lower molar was the most frequently missing tooth, while the lower canine was the least 187 lost but the most severely affected tooth (Fig. 1).

188 In the logistic regression analysis, similar results were observed in the univariable (Table 5) and 189 multivariable models (Table 6). In the multivariable analysis, smoking (OR=3.55, 95\% CI 1.80190 7.02) and older age $(\mathrm{OR}=8.70,95 \%$ CI 3.66-20.69 and $\mathrm{OR}=4.85,95 \%$ CI 2.57-9.16), for 65+ and 191 45-64 years old, respectively, were identified as risk indicators for CAL $\geq 3 \mathrm{~mm}$ (table 5).

192 Chronic periodontitis was not significantly associated with the remaining variables.

193

\section{DISCUSSION}

195 This retrospective cross-sectional study assessed the periodontal status of forwarded adult 196 subjects who sought dental treatment in a Portuguese university dental clinic, that is located in 197 the metropolitan area of Lisbon. This area has over 2.8 million inhabitants and is the largest

198 Portuguese metropolitan area ${ }^{30}$. This university dental clinic is an important reference dental 199 center in the Lisbon Region and receives patients from all social strata. The absence of complete socioeconomic data constitutes a limitation of this study. Unfortunately, over $70 \%$ of patients

201 (data not shown) refused to provide socioeconomic status information.

202 The results of this retrospective study can't be compared with previous investigations performed 203 in Portugal because in these it was applied the CPITN methodology. ${ }^{24,31-33}$ This is the first FMPE 204 protocol used in a Portuguese population and provides direct evidence for estimating periodontal 
205 status and results in a better representation of the population. ${ }^{27}$ Although FMPE methodology 206 can result in an overestimation of periodontal treatment needs among young adults, ${ }^{21}$ the partial207 mouth examination can miscalculate the prevalence of periodontitis in almost $50 \%$ of the 208 population. ${ }^{27}$ The overall results demonstrate that this referred subpopulation had a high 209 prevalence of chronic periodontitis $(79.3 \%, 95 \%$ CI $77.5-88.1 \%)$, and severe extensity of periodontal destruction among the affected subjects $(83.7 \%$, 95\% CI 81.7-85.6\%).

211 This investigation study design is not an epidemiological study per se, but rather an 212 observational study of patients who were forwarded to a periodontology consultation. Thus, we 213 were only able to estimate the prevalence and extent of our referred subpopulation. However, 214 these results underline the fact that the majority of patients attended the periodontal consultation 215 already in a state of advanced periodontal destruction and only a small percentage appeared in 216 the early stages or healthy. Still, a disturbing percentage of patients did not attend periodontal 217 consultations despite the triage referral with approximately $69 \%$ missing or unchecking the 218 appointment.

219 Regarding tooth loss, the most frequently missed teeth were the lower first molars and the less 220 missed were the lower canines, as with recent European data. ${ }^{21,22}$ Additionally, lower canines 221 and incisors were the most affected teeth with CAL and the lower molars the less. The lower 222 arch presented more periodontal destruction than the upper, and the teeth with more severe CAL 223 levels in the upper arch were the canines.

224 Concerning periodontal parameters, unlike PD, CAL severity increased with age and can be 225 related to the increase of gingival recession with aging. ${ }^{34}$ As in the literature, ${ }^{19-23}$ age was 226 confirmed in the multivariate analysis as a risk indicator for chronic periodontitis for 45-64 years 227 old $(\mathrm{OR}=4.85,95 \% \mathrm{CI} 2.57-9.16)$ and $65+$ years old $(\mathrm{OR}=8.70,95 \%$ CI 3.66-20.69). However, 228 it's important to highlight that, in the majority of CAL thresholds of subjects with the disease, 229 45-64 years old group presented worse results for prevalence of chronic periodontitis, while 65+ 230 years old group had worse levels of periodontal destruction extent.

231 Smoking was strongly associated with chronic periodontitis ( $\mathrm{OR}=3.55,95 \%$ CI 1.80-7.02). 232 Previous studies reported OR values ranging between 2 and 9 of having periodontitis. ${ }^{21-23,35-38}$ 233 Despite not accounting for lifetime smoking exposure, we stratified current smokers according to 
234 the number of cigarettes smoked although it was not significantly associated with the severity 235 and progression of the periodontal disease.

236 Several studies found that obesity was associated with an increased risk of periodontitis. ${ }^{39-41}$

237 Besides that, Suvan et al. ${ }^{42}$ concluded that overweight/obese individuals are more likely to suffer 238 from periodontitis compared to normal weight individuals. Although our results show that 239 overweight and obesity have no impact on the aggravation of periodontitis, we emphasize that 240 more than half of this subpopulation was overweight or obese, in agreement with the latest 241 national IAN-AF Food and Activity Survey. ${ }^{43}$

242 In the past, several epidemiological surveys reported that people with lower educational level 243 had higher prevalence and severity of periodontal disease. ${ }^{20,21,23,44,45}$ However, other studies have 244 indicated that this impact cannot be seen in a singular way but in a multifactorial view. ${ }^{3,46}$ Our 245 results show that despite middle education had significance in the univariable model $(\mathrm{OR}=2.09$ 246 (95\% CI 1.05-4.13), $\mathrm{p}=0.035)$, when analyzed in a multivariable model it had no impact on the 247 probability of having chronic periodontitis.

248

\section{CONCLUSION}

250 This specific subpopulation of individuals referred to periodontal examination in a university 251 dental clinic of the Lisbon region presented high prevalence and severe extent of chronic 252 periodontitis. Age and smoking were identified as risk indicators for chronic periodontitis in this 253 referred subpopulation. Within the limitations of this study, these results highlight the 254 importance of developing appropriate public health programs to educate the Portuguese 255 population about the burden of periodontal diseases.

256

257 ACKNOWLEDGMENTS

258 We thank Júlio Guilherme for his substantial support for database development and management 259 during this study. 


\section{REFERENCES}

263 1. Pihlstrom BL, Michalowicz BS, Johnson NW. Periodontal diseases. Lancet.

264 2005;366(9499):1809-20.

265 2. Darveau RP. Periodontitis: a polymicrobial disruption of host homeostasis. Nat Rev

266 Microbiol. 2010;8(7):481-90.

267 3. Albandar JM. Global risk factors and risk indicators for periodontal diseases. J

268 Periodontol 2000. 2002;29:177-206.

269 4. Albandar JM, Rams TE. Global epidemiology of periodontal diseases: an overview.

270 Periodontol 2000. 2002;29(1):7-10.

271 5. Brett PM, Zygogianni P, Griffiths GS, Tomaz M, Parkar M, D’Aiuto F, Tonetti M.

272 Functional gene polymorphisms in aggressive and chronic periodontitis. J Dent Res. $273 \quad 2005 ; 84(12): 1149-53$.

274 6. Burt B. Position paper - Epidemiology of periodontal diseases. J Periodontol. $275 \quad 2005 ; 76(8): 1406-19$.

276 7. Buset SL, Walter C, Friedmann A, Weiger R, Borgnakke WS, Zitzmann NU. Are 277 periodontal diseases really silent? A systematic review of their effect on quality of life. J $278 \quad$ Clin Periodontol. 2016;43(4):333-44.

279 8. Wang J, Lv J, Wang W, Jiang X. Alcohol consumption and risk of periodontitis: A meta$280 \quad$ analysis. J Clin Periodontol. 2016;43(7):572-83.

281 9. Keller A, Rohde JF, Raymond K, Heitmann BL. Association Between Periodontal Disease 282 and Overweight and Obesity: A Systematic Review. J Periodontol. 2015;86(6):766-76.

283 10. Preshaw PM, Alba AL, Herrera D, Jepsen S, Konstantinidis A, Makrilakis K, Taylor R. 284 Periodontitis and diabetes: a two-way relationship. Diabetologia. 2012;55(1):21-31.

285 11. Bahekar AA, Singh S, Saha S, Molnar J, Arora R. The prevalence and incidence of 286 coronary heart disease is significantly increased in periodontitis: A meta-analysis. Am 287 Heart J. 2007;154(5):830-7.

288 12. Humphrey LL, Fu R, Buckley DI, Freeman M, Helfand M. Periodontal disease and 289 coronary heart disease incidence: A systematic review and meta-analysis. J Gen Intern 
Med. 2008;23(12):2079-86.

291 13. Nibali L, Tatarakis N, Needleman I, Tu Y-K, D’Aiuto F, Rizzo M, Donos N. Association Between Metabolic Syndrome and Periodontitis: A Systematic Review and Meta-analysis. J Clin Endocrinol Metab. 2013;98(3):913-20.

294

295

296

297

298

299

300

301

302

303

14. Lafon A, Pereira B, Dufour T, Rigouby V, Giroud M, Béjot Y, Tubert-Jeannin S. Periodontal disease and stroke: A meta-analysis of cohort studies. Eur J Neurol. 2014;21(9):1155-61.

15. Leira Y, Seoane J, Blanco M, Rodríguez-Yáñez M, Takkouche B, Blanco J, Castillo J. Association between periodontitis and ischemic stroke: a systematic review and metaanalysis. Eur J Epidemiol. 2017;32(1):43-53.

16. Fuggle NR, Smith TO, Kaul A, Sofat N. Hand to mouth: A systematic review and metaanalysis of the association between rheumatoid arthritis and periodontitis. Front Immunol. 2016;7(MAR):1-10.

17. Maisonneuve AP, Amar S, Lowenfels AB. Periodontal Disease, Edentulism and Pancreatic Cancer : A Meta Analysis. Ann Oncol. 2017;28(5):985-95.

18. Papageorgiou SN, Hagner M, Nogueira AVB, Franke A, Jäger A, Deschner J. Inflammatory bowel disease and oral health: systematic review and a meta-analysis. J Clin Periodontol. 2017;44(4):382-93.

19. Bouchard P, Boutouyrie P, Mattout C, Bourgeois D. Risk assessment for severe clinical attachment loss in an adult population. J Periodontol. 2006;77(3):479-89.

20. Bourgeois D, Bouchard P, Epidemiology MC. Epidemiology of periodontal status in dentate adults in. J Periodontal Res. 2007;42(2):219-27.

21. Aimetti M, Perotto S, Castiglione A, Mariani GM, Ferrarotti F, Romano F. Prevalence of periodontitis in an adult population from an urban area in North Italy : findings from a cross-sectional epidemiological survey. J Clin Periodontol. 2015;(42):622-31.

22. Schutzhold S, Kocher T, Biffar R, Hoffmann T, Schmidt C, Micheelis W, Jordan R, Holtfreter B. Changes in prevalence of periodontitis in two German population-based studies. J Clin Periodontol. 2015;(42):121-30. 
318 23. Holde GE, Oscarson N, Trovik TA, Tillberg A, Jönsson B. Periodontitis Prevalence and 319 Severity in Adults: A Cross-Sectional Study in Norwegian Circumpolar Communities. J $320 \quad$ Periodontol. 2017;1-17.

321 24. DGS. III Estudo de Prevalência das Doenças Orais. 2015.

322 25. von Elm E, Altman DG, Egger M, Pocock SJ, Gøtzsche PC, Vandenbroucke JP. The 323 strengthening the reporting of observational studies in epidemiology (STROBE) statement: Guidelines for reporting observational studies. Int J Surg. 2014;12(12):1495-9.

26. Landry RG, Jean M. Periodontal Screening and Recording (PSR) Index: Precursors, utility and limitations in a clinical setting. Int Dent J. 2002;52(1):35-40.

327

328

329

330

331

332

333

334

335

336

337

338

339

340

341

342

343

344

27. Eke PI, Page RC, Wei L, Thornton-Evans G, Genco RJ. Update of the Case Definitions for Population-Based Surveillance of Periodontitis. J Periodontol. 2012;83(12):1449-54.

28. Susin C, Haas AN, Valle PM, Oppermann R V., Albandar JM. Prevalence and risk indicators for chronic periodontitis in adolescents and young adults in south Brazil. J Clin Periodontol. 2011;38(4):326-33.

29. Kopelman PG. Obesity as a medical problem. Nature. 2000;404(6778):635-43.

30. Área Metropolitana de Lisboa. Municípios AML [Internet]. 2018. p. (accessed 29 January 2018). Available from: https://www.aml.pt/index.php

31. Freitas E. A baseline study of periodontal conditions among youth and adults in portugal. Vol. 11, Community dental oral epidemiology. 1983. p. 250-4.

32. Marques MD, Teixeira-Pinto A, da Costa-Pereira A, Eriksen HM. Prevalence and determinants of periodontal disease in Portuguese adults: results from a multifactorial approach. Acta Odontol Scand. 2000;58(5):201-6.

33. Petersen PE, Ogawa H. The global burden of periodontal disease: Towards integration with chronic disease prevention and control. Periodontol 2000. 2012;60(1):15-39.

34. Eke PI, Dye BA, Wei L, Thornton-Evans GO, Genco RJ. Prevalence of Periodontitis in Adults in the United States: 2009 and 2010. J Dent Res. 2012;91(10):914-20.

35. Bergström J, Preber H. Tobacco Use as a Risk Factor. J Periodontol. 1994;65(5):545-50. 
345 36. Bergström J. Periodontitis and Smoking: An Evidence-Based Appraisal. J Evid Based 346 Dent Pract. 2006;6(1):33-41.

347 37. Kinane DF, Stathopoulou PG, Papapanou PN. Periodontal diseases. Nat Rev Dis Prim. $348 \quad 2017 ; 3: 17038$.

349 38. Silva-Boghossian CM, Luiz RR, Colombo AP V. Periodontal Status, Sociodemographic, 350 and Behavioral Indicators in Subjects Attending a Public Dental School in Brazil: Analysis of Clinical Attachment Loss. J Periodontol. 2009;80(12):1945-54.

39. Nishida N, Tanaka M, Hayashi N, Nagata H, Takeshita T, Nakayama K, Morimoto K, 353

40. Saito T, Shimazaki Y, Kiyohara Y, Kato I, Kubo M, Iida M, Yamashita Y. Relationship between obesity, glucose tolerance, and periodontal disease in Japanese women: The Hisayama study. J Periodontal Res. 2005;40(4):346-53.

41. Dalla Vecchia CF, Susin C, Ro CK, Oppermann RV, Albandar JM. Overweight and Obesity as Risk Indicators for Periodontitis in Adults. J Periodontol. 2005;76(10):1721-8.

42. Suvan JE, Petrie A, Nibali L, Darbar U, Rakmanee T, Donos N, D'Aiuto F. Association between overweight/obesity and increased risk of periodontitis. J Clin Periodontol.

43. IAN-AF. Inquérito Alimentar Nacional e de Atividade Física 2015-2016. 2016.

44. Albandar JM, Brunelle JA, Kingman A. Destructive periodontal disease in adults 30 years of age and older in the United States, 1988-1994. J Periodontol. 1999;70(1):13-29.

45. Krustrup U, Erik Petersen P. Periodontal conditions in 35-44 and 65-74-year-old adults in Denmark. Acta Odontol Scand. 2006;64(2):65-73.

46. Geyer S, Schneller T, Micheelis W. Social gradients and cumulative effects of income and education on dental health in the fourth German oral health study. Community Dent Oral Epidemiol. 2010;38(2):120-8. 
Figure 1

Percentage of subjects with the respective tooth present and by thresholds of CAL $(\mathrm{mm})$, at each specific position, for all teeth in all quadrants

The black lines indicate the separation by each quadrant. (Dark blue) percentage of missing teeth, (Blue) percentage of teeth with less than $3 \mathrm{~mm}$ of CAL, (pink) percentage of teeth with 3-4 mm of CAL, (yellow) percentage of teeth over $4 \mathrm{~mm}$ of $\mathrm{CAL}$

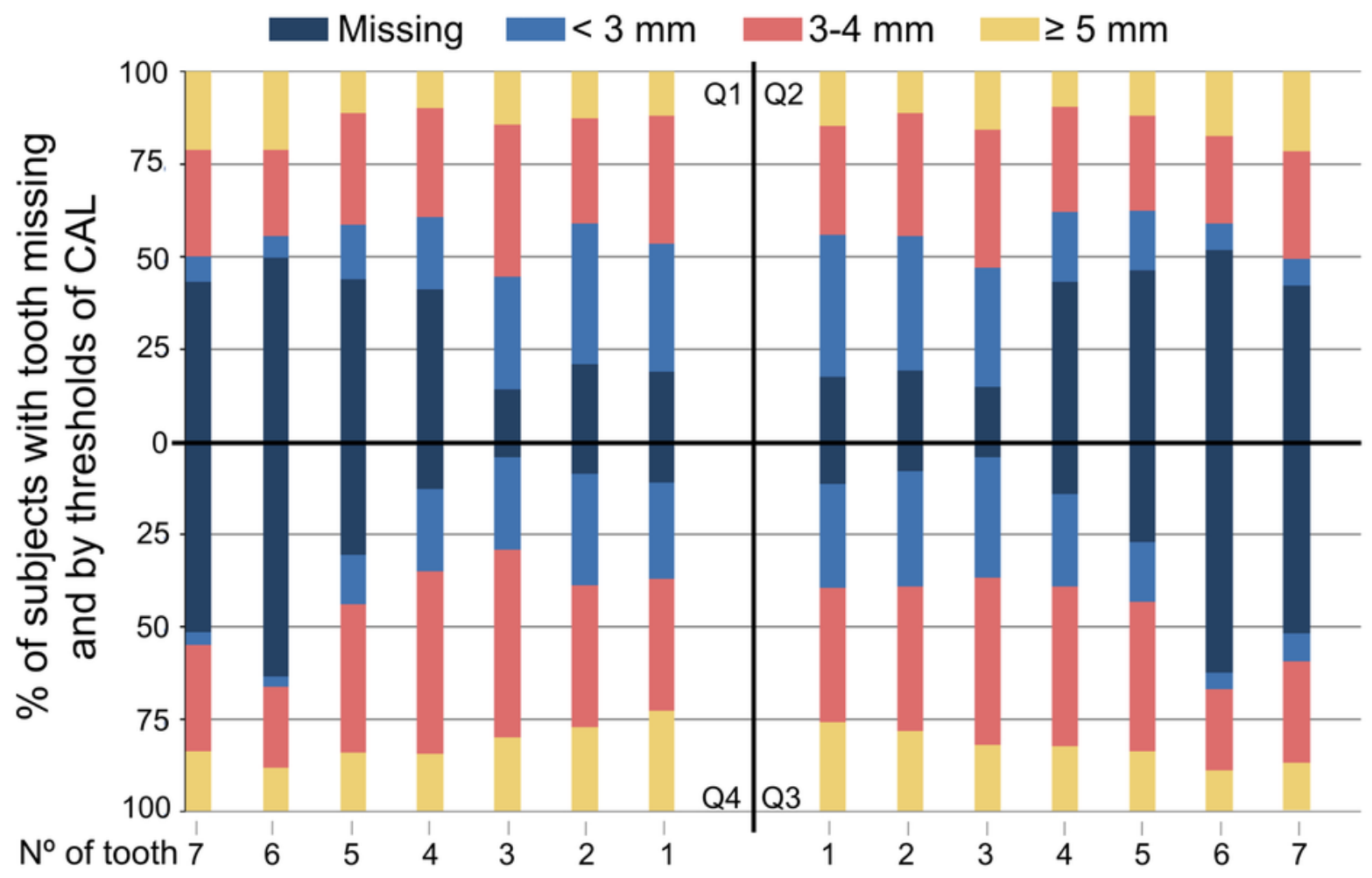




\section{Table 1 (on next page)}

Sociodemographic, behavioral, biometric and oral hygiene data $(\mathrm{N}=405)$. 
1 Table 1 - Sociodemographic, behavioural, biometric and oral hygiene data $(N=405)$

\begin{tabular}{|c|c|c|}
\hline Variable & & $n(\%)$ \\
\hline \multirow[t]{2}{*}{ Gender } & Female & $225(55.6)$ \\
\hline & Male & $180(44.4)$ \\
\hline \multirow[t]{3}{*}{ Age (years) } & $20-44$ & $90(22.2)$ \\
\hline & $45-64$ & $217(53.6)$ \\
\hline & $\geq 65$ & $98(24.2)$ \\
\hline \multirow[t]{2}{*}{ Smoking status } & Smoker & $141(34.8)$ \\
\hline & Non-smoker & $264(65.2)$ \\
\hline \multirow{2}{*}{$\begin{array}{l}\text { Active smokers } \\
\text { (cigarettes per day) }\end{array}$} & Light $(<10)$ & $41(29.1)$ \\
\hline & Medium (10-20) & $93(66.0)$ \\
\hline$(n=141)$ & Heavy $(>20)$ & $7(5.0)$ \\
\hline \multirow[t]{3}{*}{ Education } & Elementary & $157(38.8)$ \\
\hline & Middle & $155(38.3)$ \\
\hline & Higher & $93(23.0)$ \\
\hline \multirow[t]{3}{*}{ Employment status } & Employed & $210(51.9)$ \\
\hline & Unemployed & $63(15.6)$ \\
\hline & Retired & $132(32.6)$ \\
\hline \multirow[t]{2}{*}{ BMI $\left(\mathrm{kg} / \mathrm{m}^{2}\right)$} & $<18.5$ & $5(1.2)$ \\
\hline & $18.5-24.9$ & $162(40.0)$ \\
\hline
\end{tabular}




\begin{tabular}{|c|c|c|}
\hline & $25.0-29.9$ & $159(39.3)$ \\
\hline & $\geq 30$ & 79 (19.5) \\
\hline \multirow[t]{5}{*}{ Last dental visit } & $<1$ year & $185(45.7)$ \\
\hline & 1 - 2 years & $57(14.1)$ \\
\hline & $3-4$ years & $75(18.5)$ \\
\hline & $\geq 5$ years & $83(20.5)$ \\
\hline & Never & $5(1.2)$ \\
\hline \multirow[t]{5}{*}{ Consultation motive } & Routine & $125(30.9)$ \\
\hline & Aesthetics & $35(8.6)$ \\
\hline & Pain & $73(18.0)$ \\
\hline & Functional & $157(38.8)$ \\
\hline & Other & $15(3.7)$ \\
\hline \multirow[t]{2}{*}{ Dental floss usage } & Yes & $141(34.8)$ \\
\hline & No & $264(65.2)$ \\
\hline \multirow[t]{3}{*}{ Toothbrush frequency } & 2 - 3 times / daily & $313(77.3)$ \\
\hline & 1 time / daily & $75(18.5)$ \\
\hline & 2- 6 times / weekly & $17(4.2)$ \\
\hline
\end{tabular}




\section{Table 2 (on next page)}

Periodontal clinical data (presented as mean \pm standard deviation) as a function of gender, age and smoking status $(\mathrm{N}=405)$. 
1 Table 2 - Periodontal clinical data (presented as mean \pm standard deviation) as a function of gender, age and smoking status $(N=$ 405).

3

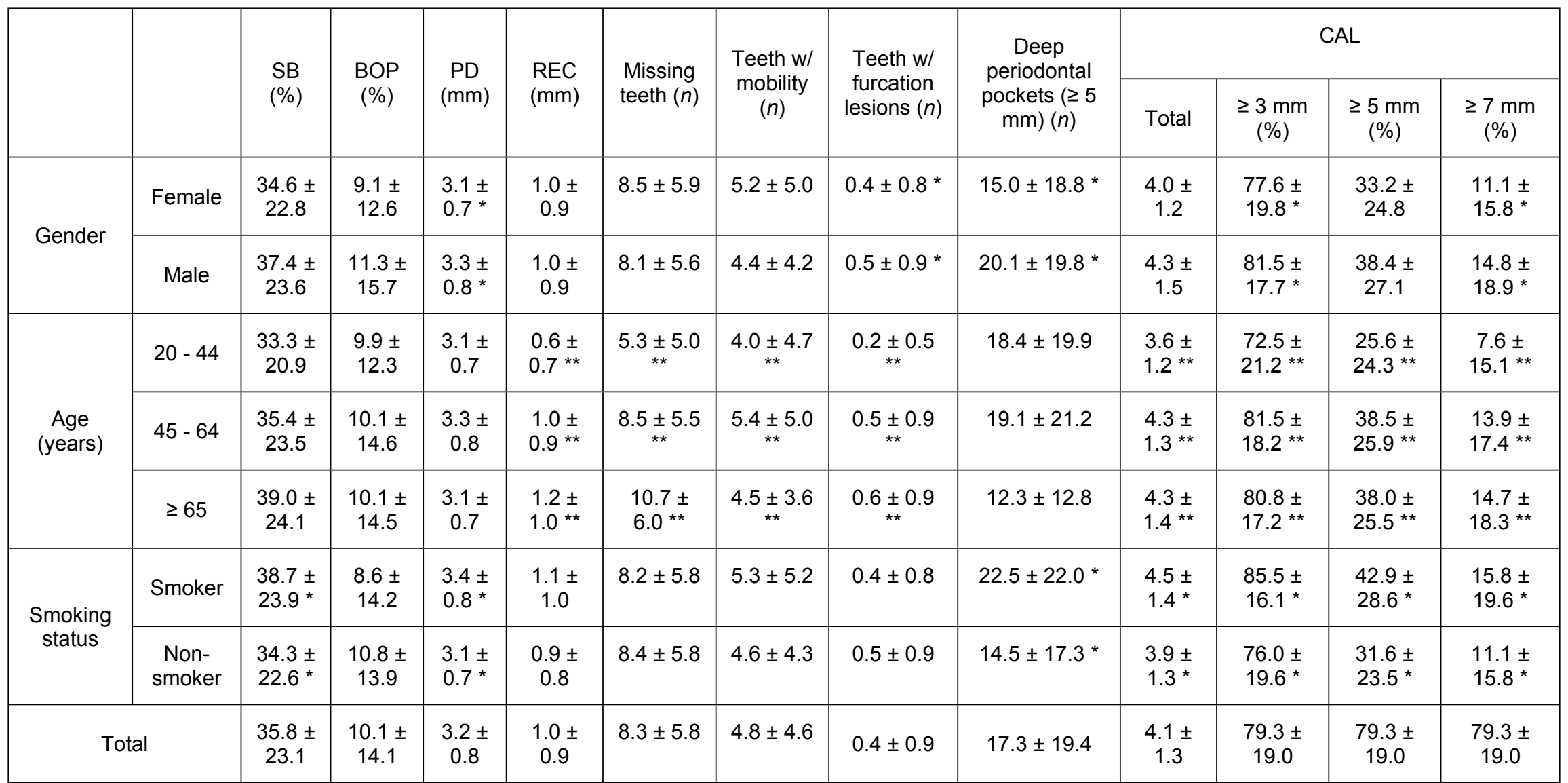

4 SB - Supragingival Biofilm, BOP - Bleeding on Probing, PD - Pocket Depth, REC - Recession, CAL - Clinical Attachment Loss

5 * Mann-Whitney test $(p<0.05),{ }^{* *}$ Kruskal-Wallis test $(p<0.05)$

6

7 


\section{Table 3(on next page)}

Percentage of patients, with $95 \%$ confidence interval $(95 \% \mathrm{Cl})$, by threshold of CAL $(\mathrm{mm})$, severity and age group (years). 
1 Table 3 - Percentage of patients, with 95\% confidence interval (95\% Cl), by threshold of CAL (mm), severity and age group (years)

\begin{tabular}{|c|c|c|c|c|c|c|c|c|c|c|c|c|c|c|c|c|}
\hline \multirow{3}{*}{$\begin{array}{l}\mathrm{CAL} \\
(\mathrm{mm})\end{array}$} & \multicolumn{8}{|c|}{ Subjects with chronic periodontitis } & \multicolumn{8}{|c|}{ All subjects } \\
\hline & \multicolumn{2}{|c|}{$\begin{array}{c}20-44 \\
(n=59)\end{array}$} & \multicolumn{2}{|c|}{$\begin{array}{c}45-64 \\
(n=190)\end{array}$} & \multicolumn{2}{|c|}{$\begin{array}{c}\geq 65 \\
(n=89)\end{array}$} & \multicolumn{2}{|c|}{$\begin{array}{c}\text { Total } \\
(n=338)\end{array}$} & \multicolumn{2}{|c|}{$\begin{array}{c}20-44 \\
(n=90)\end{array}$} & \multicolumn{2}{|c|}{$\begin{array}{c}45-64 \\
(n=217)\end{array}$} & \multicolumn{2}{|c|}{$\begin{array}{c}\geq 65 \\
(n=98)\end{array}$} & \multicolumn{2}{|c|}{$\begin{array}{c}\text { Total } \\
(\mathrm{n}=405)\end{array}$} \\
\hline & $\%$ & $95 \% \mathrm{Cl}$ & $\%$ & $95 \% \mathrm{Cl}$ & $\%$ & $95 \% \mathrm{Cl}$ & $\%$ & $95 \% \mathrm{Cl}$ & $\%$ & $95 \% \mathrm{Cl}$ & $\%$ & $95 \% \mathrm{Cl}$ & $\%$ & $95 \% \mathrm{Cl}$ & $\%$ & $95 \% \mathrm{Cl}$ \\
\hline
\end{tabular}

Prevalence (patients)

\begin{tabular}{|c|c|c|c|c|c|c|c|c|c|c|c|c|c|c|c|c|}
\hline$\geq 3$ & 100 & $100.0-100.0$ & 100 & $100.0-100.0$ & 100 & $100.0-100.0$ & 100 & $100.0-100.0$ & 65.6 & $56.1-75.1$ & 87.6 & $83.5-91.7$ & 90.8 & $85.3-96.3$ & 83.5 & 80.4-86.6 \\
\hline$\geq 4$ & 42.4 & $30.0-54.8$ & 62.1 & $55.7-68.6$ & 59.6 & 49.7-69.5 & 58.0 & $53.4-62.6$ & 27.8 & $18.8-36.8$ & 54.4 & $48.3-60.5$ & 54.1 & $44.6-63.6$ & 48.4 & $44.2-52.6$ \\
\hline$\geq 5$ & 20.3 & $10.2-30.4$ & 30.0 & 23.9-36.1 & 25.8 & $17.0-34.6$ & 15.4 & $12.0-18.8$ & 13.3 & $6.5-20.1$ & 26.3 & $20.9-31.7$ & 23.5 & 15.4-31.6 & 12.8 & $10.0-15.6$ \\
\hline$\geq 6$ & 8.5 & 1.515 .5 & 13.2 & 8.7-17.7 & 12.4 & $5.8-19.0$ & 12.1 & $9.0-15.2$ & 5.6 & $1.0-10.2$ & 11.5 & $7.6-15.4$ & 11.2 & $5.2-17.2$ & 10.1 & 7.6-12.6 \\
\hline$\geq 7$ & 5.1 & $0.0-10.6$ & 0.5 & $0.0-1.4$ & 5.6 & $1.0-10.2$ & 5.0 & 3.0-7.1 & 3.3 & $0.0-6.9$ & 4.1 & $1.7-6.5$ & 5.1 & $0.9-9.3$ & 4.2 & $2.5-5.9$ \\
\hline
\end{tabular}

Cl- Confidence Interval, CAL - Clinical Attachment Loss 


\section{Table 4 (on next page)}

Percentage, with $95 \%$ confidence interval $(95 \% \mathrm{Cl})$, of sites (prevalence) and affected teeth (extent), by threshold of CAL ( $\mathrm{mm})$, severity and age group (years). 
1 Table 4 - Percentage, with 95\% confidence interval $(95 \% \mathrm{Cl})$, of sites (prevalence) and affected teeth (extent), by threshold of CAL 2 (mm), severity and age group (years)

3

\begin{tabular}{|c|c|c|c|c|c|c|c|c|c|c|c|c|c|c|c|c|}
\hline \multirow{3}{*}{$\begin{array}{l}\text { CAL } \\
(\mathrm{mm})\end{array}$} & \multicolumn{8}{|c|}{ Subjects with chronic periodontitis } & \multicolumn{8}{|c|}{ All subjects } \\
\hline & \multicolumn{2}{|c|}{$\begin{array}{l}20-44 \\
(n=59)\end{array}$} & \multicolumn{2}{|c|}{$\begin{array}{c}45-64 \\
(n=190)\end{array}$} & \multicolumn{2}{|c|}{$\begin{array}{c}\geq 65 \\
(n=89)\end{array}$} & \multicolumn{2}{|c|}{$\begin{array}{c}\text { Total } \\
(\mathrm{n}=338)\end{array}$} & \multicolumn{2}{|c|}{$\begin{array}{c}20-44 \\
(\mathrm{n}=90)\end{array}$} & \multicolumn{2}{|c|}{$\begin{array}{c}45-64 \\
(n=217)\end{array}$} & \multicolumn{2}{|c|}{$\begin{array}{c}\geq 65 \\
(n=98)\end{array}$} & \multicolumn{2}{|c|}{$\begin{array}{c}\text { Total } \\
(\mathrm{n}=405)\end{array}$} \\
\hline & $\%$ & $95 \% \mathrm{Cl}$ & $\%$ & $95 \% \mathrm{Cl}$ & $\%$ & $95 \% \mathrm{Cl}$ & $\%$ & $95 \% \mathrm{Cl}$ & $\%$ & $95 \% \mathrm{Cl}$ & $\%$ & $95 \% \mathrm{Cl}$ & $\%$ & $95 \% \mathrm{Cl}$ & $\%$ & $95 \% \mathrm{Cl}$ \\
\hline
\end{tabular}

\section{Prevalence (sites)}

\begin{tabular}{|c|c|c|c|c|c|c|c|c|c|c|c|c|c|c|c|c|}
\hline$\geq 3$ & 85.3 & $82.7-87.4$ & 86.9 & $85.3-88.5$ & 84.4 & $81.7-87.1$ & 86.0 & $84.7-87.2$ & 72.5 & $68.0-76.9$ & 81.5 & $79.0-83.9$ & 80.8 & $77.4-84.2$ & 79.3 & $77.5-81.2$ \\
\hline$\geq 4$ & 57.0 & $51.6-62.4$ & 61.3 & $58.1-64.5$ & 59.2 & $54.5-63.9$ & 60.0 & $57.6-62.4$ & 41.5 & $35.8-47.1$ & 55.2 & $51.7-58.7$ & 55.3 & $50.2-60.4$ & 52.2 & $49.6-54.8$ \\
\hline$\geq 5$ & 36.9 & $30.7-43.0$ & 43.3 & $39.8-46.8$ & 41.2 & $36.1-46.3$ & 41.6 & $39.0-44.2$ & 25.6 & $20.5-30.7$ & 38.5 & $35.1-42.0$ & 38.0 & $33.0-43.1$ & 35.5 & $33.0-38.1$ \\
\hline$\geq 6$ & 21.9 & $16.4-27.4$ & 27.7 & $24.5-30.9$ & 26.8 & $22.2-31.4$ & 26.5 & $24.1-28.9$ & 14.7 & $10.6-18.8$ & 24.5 & $21.5-27.5$ & 24.6 & 20.1-29.1 & 22.4 & $20.2-24.5$ \\
\hline$\geq 7$ & 11.7 & $7.0-16.4$ & 15.8 & $13.2-18.3$ & 16.1 & $12.2-20.1$ & 15.2 & $13.2-17.1$ & 7.6 & $4.4-10.8$ & 13.9 & 11.6-16.2 & 14.7 & $11.0-18.4$ & 12.7 & $11.0-14.4$ \\
\hline
\end{tabular}

Extent (affected teeth)

\begin{tabular}{|c|c|c|c|c|c|c|c|c|c|c|c|c|c|c|c|c|}
\hline$\geq 3$ & 82.1 & $77.6-86.6$ & 85.0 & $82.5-87.7$ & 81.7 & $77.6-85.7$ & 83.7 & $81.7-85.6$ & 62.1 & $55.5-68.7$ & 77.3 & $73.7-80.9$ & 77.0 & $72.3-81.7$ & 73.9 & $71.1-76.6$ \\
\hline$\geq 4$ & 49.3 & $41.9-56.7$ & 56.1 & $51.9-60.3$ & 54.0 & $47.8-60.2$ & 54.4 & $51.3-57.5$ & 33.5 & 27.1-39.9 & 49.8 & $45.5-54.0$ & 49.5 & $43.2-55.8$ & 46.1 & $43.0-49.2$ \\
\hline$\geq 5$ & 25.0 & $17.8-32.1$ & 33.4 & $29.2-37.6$ & 33.8 & $27.8-39.8$ & 32.1 & $29.0-35.2$ & 16.0 & $10.9-21.2$ & 29.5 & $25.6-33.4$ & 30.8 & $25.0-36.5$ & 26.8 & $24.0-29.6$ \\
\hline$\geq 6$ & 12.3 & $6.8-17.8$ & 18.6 & $15.2-22.0$ & 19.5 & $14.3-24.7$ & 17.8 & $15.2-20.3$ & 7.9 & $4.2-11.6$ & 16.3 & $13.2-19.3$ & 17.7 & $12.8-22.6$ & 14.7 & $12.6-16.9$ \\
\hline$\geq 7$ & 5.8 & $2.1-9.4$ & 10.0 & $7.5-12.4$ & 9.9 & $5.8-14.1$ & 9.2 & $7.4-11.1$ & 3.7 & $1.3-6.0$ & 8.7 & $6.5-10.8$ & 9.0 & $5.2-12.8$ & 7.6 & $6.1-9.2$ \\
\hline
\end{tabular}

4 Cl- Confidence Interval, CAL - Clinical Attachment Loss

5

6

7 


\section{Table 5 (on next page)}

Univariate logistic regression analysis of sociodemographic, behavioural, anthropometric and oral hygiene variables for the outcome variable CAL $\geq 3 \mathrm{~mm}$ ( $N=$ 405) 
1 Table 5 - Univariate logistic regression analysis of sociodemographic, behavioural, anthropometric and oral hygiene variables for the 2 outcome variable $\mathrm{CAL} \geq 3 \mathrm{~mm}(N=405)$

\begin{tabular}{|c|c|c|c|}
\hline Predictor variables & & OR $(95 \% \mathrm{CI})$ & $p$ \\
\hline \multirow{2}{*}{ Gender } & Female & 1 & - \\
\hline & Male & $1.32(0.77-2.26)$ & 0.310 \\
\hline \multirow[t]{4}{*}{ Age (years) } & & & $<0.001$ \\
\hline & $20-44$ & 1 & - \\
\hline & $45-64$ & $3.70(2.04-6.69)$ & $<0.001$ \\
\hline & $\geq 65$ & $5.20(2.31-11.70)$ & $<0.001$ \\
\hline \multirow{2}{*}{ Smoking status } & Smoker & $2.06(1.11-3.81)$ & 0.021 \\
\hline & Non-smoker & 1 & - \\
\hline \multirow[t]{4}{*}{ Education (years) } & & & 0.107 \\
\hline & $1-4$ & $1.40(0.74-2.66)$ & 0.298 \\
\hline & $5-12$ & $2.09(1.05-4.13)$ & 0.035 \\
\hline & $>12$ & 1 & - \\
\hline \multirow[t]{4}{*}{ Employment status } & & & 0.246 \\
\hline & Employed & 1 & - \\
\hline & Unemployed & $1.67(0.74-3.77)$ & 0.219 \\
\hline & Retired & $1.54(0.84-2.81)$ & 0.163 \\
\hline \multirow[t]{2}{*}{ BMI (kg/m²) } & & & 0.699 \\
\hline & $<18.5$ & 1 & - \\
\hline
\end{tabular}




\begin{tabular}{|c|c|c|c|}
\hline & $18.5-24.9$ & $1.06(0.11-9.79)$ & 0.961 \\
\hline & $25.0-29.9$ & $1.48(0.16-13.82)$ & 0.732 \\
\hline & $\geq 30$ & $1.40(0.14-13.59)$ & 0.774 \\
\hline \multirow[t]{6}{*}{ Last dental visit } & & & 0.026 \\
\hline & $<1$ year & 1 & - \\
\hline & $1-2$ years & $1.39(0.54-3.57)$ & 0.493 \\
\hline & $3-4$ years & $0.42(0.22-0.81)$ & 0.009 \\
\hline & $>=5$ years & $0.97(0.46-2.03)$ & 0.930 \\
\hline & Never & $0.24(0.04-1.54)$ & 0.134 \\
\hline \multirow[t]{6}{*}{ Consultation motive } & & & 0.806 \\
\hline & Routine & 1 & - \\
\hline & Aesthetics & $0.72(0.29-1.80)$ & 0.483 \\
\hline & Pain & $1.09(0.50-2.35)$ & 0.834 \\
\hline & Functional & $1.24(0.66-2.36)$ & 0.502 \\
\hline & Other & $1.39(0.29-6.60)$ & 0.680 \\
\hline \multirow{2}{*}{ Dental floss use } & Yes & 1 & - \\
\hline & No & $1.66(0.97-2.82)$ & 0.063 \\
\hline \multirow[t]{4}{*}{ Toothbrush frequency } & & & 0.803 \\
\hline & 2 - 3 times / daily & 1 & - \\
\hline & 1 time / daily & $1.27(0.63-2.56)$ & 0.508 \\
\hline & 2- 6 times / weekly & - & 0.998 \\
\hline
\end{tabular}




\section{Table 6(on next page)}

Multivariate logistic regression analysis (final reduced model) $(*)$ for the outcome variable $C A L \geq 3 \mathrm{~mm}(\mathrm{~N}=405)$. 
2 Table 6 - Multivariate logistic regression analysis (final reduced model) $\left(^{*}\right)$ for the outcome variable $C A L \geq 3 \mathrm{~mm}(N=405)$

\begin{tabular}{|c|c|c|c|}
\hline \multicolumn{2}{|c|}{ Predictor variables } & \multicolumn{2}{c|}{ CAL $\geq 3 \mathrm{~mm}$} \\
\cline { 3 - 4 } & OR $(95 \% \mathrm{Cl})$ & $p$ \\
\hline \multirow{3}{*}{ Age (years) } & $20-44$ & 1 & - \\
\cline { 2 - 4 } & $45-64$ & $4.85(2.57-9.16)$ & $<0.001$ \\
\cline { 2 - 4 } & $\geq 65$ & $8.70(3.66-20.69)$ & $<0.001$ \\
\hline \multirow{3}{*}{ Smoking status } & Non-smoker & 1 & - \\
\cline { 2 - 4 } & Smoker & $3.55(1.80-7.02)$ & $<0.001$ \\
\hline
\end{tabular}

4

Cl- Confidence Interval, OR - Odds Ratio, CAL - Clinical Attachment Loss

$\left(^{*}\right)$ The model was statistically significant, $\chi^{2}(3)=39.507, p<0.001$, explained $15.7 \%$ (Nagelkerke $R^{2}$ ) of the variance and correctly classified $83.5 \%$ of cases. 